\title{
A BOTTOM-UP QUANTITATIVE MECHANISM FOR UPDATING THE SELF- MODIFICATION PARAMETERS IN THE SLEUTH MODEL
}

\author{
F. Liu ${ }^{1,2}$,W. L. Sun ${ }^{1}$ \\ ${ }^{1}$ Institute of Geomatics and Urban Spatial Information, Beijing University of Civil Engineering and \\ Architecture, Beijing, China -1f@bucea.edu.cn; 2108521518026@stu.bucea.edu.cn
}

${ }^{2}$ Department of Geography, University of Wisconsin-Milwaukee, Milwaukee, United States of America - liu297@uwm.edu

\section{Commission III, WG III/7}

KEY WORDS: Bottom-Up; SLEUTH; Sensitivity Analysis; urban expansion.

\begin{abstract}
:
Generally, the default Self-Modification Parameters (SMPs) values, rather than the proper SMPs parameters group, has been applied in the SLEUTH model. However, various pre-setting of SMPs will simulate different morphology and structure of urban sprawl. The study is intended to propose a practical tool for the quantification response to model input variables on modelling complex urban systems. In this research, the parameter weight sorting job has been carried out to provide an adjusting priority experience. Besides, the model output imagery indices were used to describe the morphology, distribution of the urban prediction sprawl and the correlationship with urban road network effectively. Finally, the adjusting factors have been calculated with AHP method. The work helps geographers to determine how to make further use of the inner forward transmission mechanism on the SLEUTH model for further improving it in performance.
\end{abstract}

\section{INSTRUCTION}

With the prosperity of urbanization, the development of predicting tools of urban land system has been promoted(Smith, 2019). As one popular representative, the SLEUTH (slope, land-use, exclusion, urban extent, transportation and hill-shade) model has been used for simulating complex urban systems with historic remote sensing data (Chaudhuri and Clarke, 2013; Clarke, 2008; Feng et al., 2012). Characterized by the "selfmodification" rules and a "brute force calibration" technique, which could narrow down the range of prediction behaviour parameters automatically, the SLEUTH model predict the land use in future (Feng, 2012). However, the morphology and structure of urban sprawl vary greatly because the selfmodification parameters (SMPs) settings (Feng et al., 2012; Jafarnezhad et al., 2016). Meanwhile, the model's behaviour is affected by its other controlling parameters, such as Working Grids, Random Number Seed, Monte Carlo Iteration, Excluded Map and Calibration Parameters setup(Chaudhuri and Foley, 2019; Feng et al., 2012). Little detailed research is available about the transmission mechanism for the proper sleuth model self-modification parameters setting yet (Saxena and Jat, 2019). A sensitivity analysis, which has been used in Cellular Automata model, is also a useful tool to explore the quantitative transmission mechanism for urbanization model research. Lessons from sensitivity analysis of a Cellular Automata urban land use model is also the sensitivity analysis for stable performance of SLEUTH model.

In this paper, the SMPs setting is updated with the images predicted, rather than the default ones. Besides, a set of image metrics proposed will be used to quantitatively evaluate the differences among image simulation.

\section{RESEARCH BACKGROUND}

Sensitivity analysis (SA) represents an important step in improving the understanding and use of land-use change prediction models (Hewitt and Diaz-Pacheco, 2017; Kocabas and Dragicevic, 2006). The inaccurate results and model uncertainty might be attributed to the structure and nature of the model. SA is the only critical tool for quantifying the response of model input variables for modelling complex urban systems and avoiding unusually high or low growth rates of urban expansion. There are many different SA approaches. Overall, they can be categorized into two groups: local SA and global SA (Hewitt and Diaz-Pacheco, 2017; Schouten et al., 2014; Zoras et al., 2007). The local SA explores the changes of model responses by varying one parameter while keeping the other parameters constant. The simplest and most common approach is differential SA (DSA), which uses partial derivatives or finite differences of parameters at a fixed step as the measure of parametric sensitivity (Song et al., 2015). On the other hand, the global SA examines the changes in the model responses by varying all parameters at the same time, allowing them to provide robust measures in the presence of nonlinearity and interactions among the parameters; thus, they are generally preferred due to their global properties (Saltelli et al., 2008). The generalized SA (GSA) method is one of the global SA methods that have been designed to overcome the limitations of local SA methods (Park et al., 2016). A version of the GSA method, the Generalized Likelihood Uncertainty Estimation (GLUE) method, was developed by Beven \& Binley (1992) (Beven and Binley, 1992). GSA is simple to implement and can work with different pseudo-likelihoods (i.e., goodness of fit) measures (Beven, 2011), but it is computationally inefficient.

Especially speaking, One-at-a-time (OAT) approaches have recently gained popularity because they offer the most representative local sensitivity measures while maintaining computational efficiency. In sum, the complexity of these

\footnotetext{
* Corresponding author: Fang Liu (lf@bucea.edu.cn)
} 
problems highlights the need to understand the sources and range of uncertainty associated with different aspects of the modelling process (Shafizadeh-Moghadam et al., 2017; Xu and Zhang, 2013). It is easy to implement, computationally inexpensive and useful for providing a glimpse at the model behaviour. Recently, Hewitt and Diaz-Pacheco (2017) investigated the influence of the stochastic factor, the influence of scale and cell size to the simulation results (Hewitt and DiazPacheco, 2017). Saxena and Jat (2018) studied the SLEUTH model sensitivity to self-modifying parameter and identified the influence of self-modifying parameters on fragmented urban growth (Jat et al., 2017). Then, the authors above optimized the slope layer, identified the weight of factors for land suitability (Saxena and Jat, 2020). However, when it comes to single factor sensitivity analysis of SMPs, where the default SMPs setting are usually adopted, rather than the proper ones, it has been seldom discussed.

But as all know, proper SMPs values will produce healthy predicted images with OAT SA method, including the morphology and structure of urban sprawl. In this paper, two issues would be dealt with: 1) One scheme which probes the response relationship between independent variables and dependent variables will be proposed. 2) with the knowledge rules, the input parameters strategy is adjusted according to the trend and size of the simulation to make sure the predicted urban expansion close to the real one. These questions are addressed with the ample image data from SLEUTH3.0 beta_p01 LINUX, released 6/2005 and sponsored by Project Gigalopolis (USGS and UCSB, 2005).

\section{MATERIALS AND METHODS}

\subsection{Data}

The tested object is the demo200 image package along with "SLEUTH3.0beta_p01 linux", which is dependent on model input parameters. Figure 1 illustrates the input maps in $1 \mathrm{~m}$ resolution with size of 200 pixels $\times 200$ pixels.
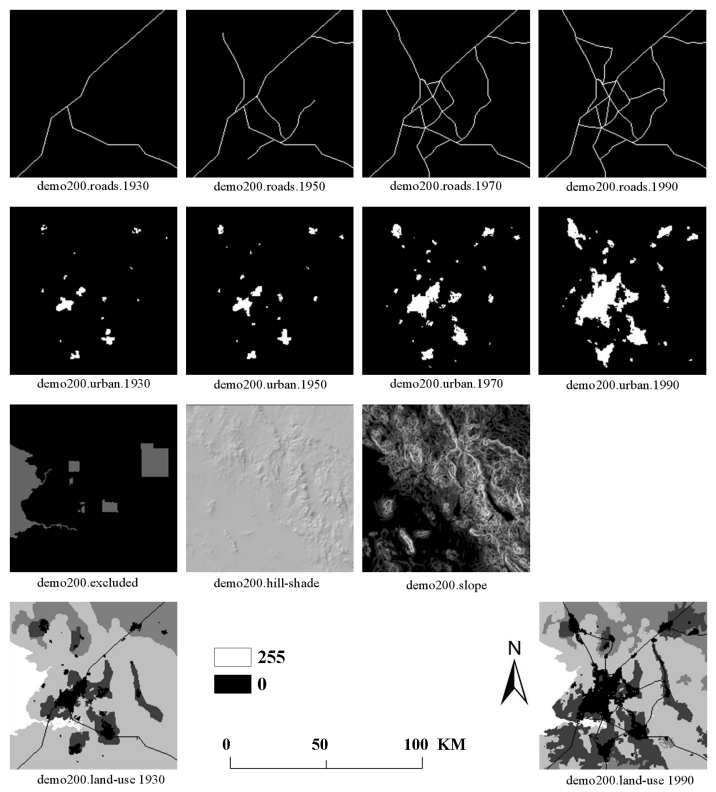

Figure 1 . The input maps of demo200 with a size of $200 \times 200$

Figure 1 illustrates the construction of an SLEUTH model input image. In figure 1, it consists of six types of data: slope, landuse, exclude, urban, roads and hill-shade.

\subsection{Parameters and Scheme of Sensitivity Analysis}

The model self-modification parameters (SMPs) combinations includes seven parameters: road_grav_sensitivity, slope_sensitivity, critical_low, critical_high, critical_slope, boom and bust. These default value settings govern the processing, which determine the urban simulation imagery.

The SA research process has been divided into three procedures (in Figure 2): first, an minor increments of SMPs has caused the corresponding change of the prediction best fit values (PBFs), which is called stage (1): $\mathrm{SMP}+\boldsymbol{\delta} \rightarrow \mathrm{PBF}+\boldsymbol{\Delta}$. However, there is no distinct reflection of prediction accuracy. second, the corresponding alterations of predicted images appear, which is called stage (2) : $\mathrm{SMP}+\boldsymbol{\delta} \rightarrow$ Final results $+\boldsymbol{\Theta}$. Third, a new alteration value $\boldsymbol{\delta}^{\prime}$ is calculated according to the parameterresult record database established before, which is called stage (3), namely, the feedback mechanism, like the following.

stage (1): the response of processing results to the Initialization parameters variation has been recorded and a new rule was established.

stage (2): the response of final results to the Initialization parameters variation has been recorded and the above rule was supplemented.

stage (3): an important feedback mechanism from the rules for monitoring the control governance was extracted.

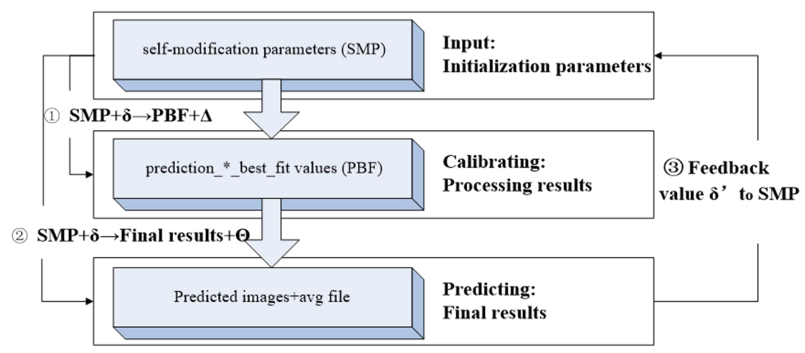

Figure 2. Work-flow chart of the SLEUTH Simulation framework

Note: (1) SMPs are the initial default value setting and are expressed as $\left\{\boldsymbol{N}_{i}, \quad \boldsymbol{i}=1,2,3,4,5,6,7\right\}$. They are $\boldsymbol{N}_{\boldsymbol{l}}$ : road_grav_sensitivity, $N_{2}$ : slope_sensitivity, $N_{3}$ : critical_low, $N_{4}$ : critical_high, $N_{5}$ : critical_slope, $N_{6}$ : boom and $N_{7}$ : bust, respectively. One suite of parameters is set with only one $\delta$ alteration added upon one $\mathrm{Ni}$ parameter, expressed as $\left\{\boldsymbol{N}_{\boldsymbol{l}}\right.$, $\left.N_{2}, \ldots, N_{i}+\delta_{i}, \ldots N_{7}\right\}$. There are 65 suites of parameter sets in total, as one alteration is added to one SMP each time.

(2) PBFs are the processing record by altering the SMP parameter set suite, which are expressed as $\left\{\boldsymbol{G}_{\boldsymbol{k}}, \boldsymbol{k}=1,2,3,4,5\right\}$. They are $\boldsymbol{G}_{\boldsymbol{1}}$ : prediction_diffusion_best_fit, $\boldsymbol{G}_{2}$ : prediction_spread_best_fit, $\boldsymbol{G}_{3}$ : prediction_breed_best_fit, $\boldsymbol{G}_{4}$ : prediction_slope_resistance_best_fit, prediction_road_gravity_best_fit. PBFs, which are supposed to predict the final images and record, are closely related to SMPs.

This paper tends to explore a model with a set of different initial states, while the initial setup of the model used parameters $\left\{N_{i}\right\}$ with default values. The initial model parameters are default values along with SLEUTH code, supplied by the original 
designers. They are $N_{1}$ : road_grav_sensitivity $=0.01, \quad N_{2}$ : slope_sensitivity $=0.1, \quad N_{3}$ : $\quad$ critical_low $=0.97, \quad N_{4}$ : critical_high=1.03, $N_{5}$ : critical_slope $=15, \bar{N}_{6}$ : boom=1.01 and $N_{7}$ : bust $=0.09$.

The aim of variation in the design is to make a clear Bottom-Up quantitative model transmission mechanism. Based on the OAT SA method, this paper measures the systematic response to each independent parameter variation with a variety of sizes and tendencies. As $\boldsymbol{N}_{3}$ and $\boldsymbol{N}_{4}$, are divided into one group, which are widened or narrowed simultaneously. There are altogether six groups of input controlling parameters, and 65 experiment suites are recorded. One parameter suite has only one $\delta$ alteration to one parameter. The minimum step size is set to 0.02 or 0.2 (CRITICAL_SLOPE only), while the range of change is from -100 to 100 times. The output response to parameters variation is shown in Figure 3.

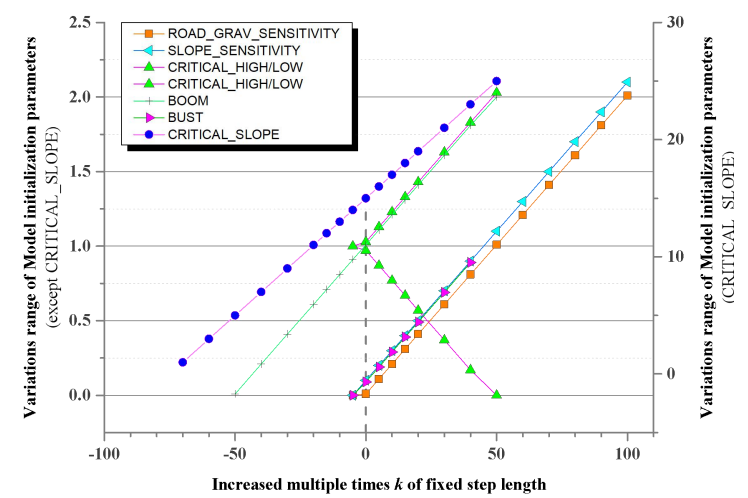

Figure 3. Variation of model initialization parameters (in chart form). The $\mathbf{X}$ axis stands for an increment of multiple times $\mathrm{k}$ of the initial parameter, and the $\mathrm{Y}$ axis is a double axis, standing for the variation range of model initialization parameters, where the left axis indicates the variation range of all parameters except for critical_slope and the right axis indicates the variation range of critical_slope.

The final results include the Predicted images and avg files, which is also the record of a model input parameters set SMPs variation. Prediction images are land-use charts from 1991 to 2010 , for a total of twenty years. Considering too many images, $20 * 65$, simplified metrics are used to measure the charts: $\boldsymbol{M}_{1}$ : Urban expansion directional distribution, $\boldsymbol{M}_{2}$ : Urban clusters aggregation, $\boldsymbol{M}_{3}$ : Urban area proportion, and $\boldsymbol{M}_{4}$ : Roads' correlation with urban area. The alteration on the final chart are expressed as $\left\{\boldsymbol{M}_{1}+\boldsymbol{\Theta}_{1}, \boldsymbol{M}_{2}+\boldsymbol{\Theta}_{2}, \ldots, \boldsymbol{M}_{j}+\boldsymbol{\Theta}_{j}, \ldots \boldsymbol{M}_{4}+\boldsymbol{\Theta}_{4}\right\}$. For avg files, they are coefficient records which decide how the growth rules are applied year by year.

\subsection{Two derivatives for Evaluating the Simulation}

When SA information mining is dealt with, two derivatives from the Absolute Value are proposed: the first-order difference to a fixed reference and the first-order difference to a relative reference (formulas (1)-(2)).The first-order difference to a fixed reference is applied to analyse the differences ( 4 ) between the output predicted maps/data and standard reference map, but the latter does not change the control parameters set. Additionally, the first-order difference to a shifting reference is used to evaluate the differences (A) between two consecutive output predicted maps/data. To unify the rate of output variation, the above mentioned differences $(\Delta)$ have both been divided by the change of the input parameter $(\boldsymbol{\delta})$. The expression formulas are:

$$
\begin{gathered}
P B F_{1}^{k}=\frac{\Delta_{r e f+\delta}^{k}-\Delta_{r e f}^{k}}{|\delta|} \\
P B F_{2}^{k}=\frac{\Delta_{r e f+\delta}^{k}-\Delta_{r e f+\delta^{\prime}}^{k}}{\left|\delta-\delta^{\prime}\right|}
\end{gathered}
$$

where, $\mathrm{SMP}+\boldsymbol{\delta}$ is the expression of $\left\{\boldsymbol{N}_{1}, \boldsymbol{N}_{2}, \ldots, \boldsymbol{N}_{i}+\boldsymbol{\delta}_{\mathrm{i}}, \ldots \boldsymbol{N}_{7}\right\}$; $\mathrm{PBF}+\boldsymbol{\Delta}$ is the expression of $\left\{\boldsymbol{G}_{1}+\boldsymbol{\Delta}_{1}, \boldsymbol{G}_{2}+\boldsymbol{\Delta}_{2}, \ldots\right.$, $\left.\boldsymbol{G}_{\boldsymbol{k}}+\boldsymbol{\Delta}_{k} \ldots \boldsymbol{G}_{5}+\boldsymbol{\Delta}_{5}\right\} ; \boldsymbol{i}$ is the subscript of $\left\{\boldsymbol{N}_{\boldsymbol{i}}, \boldsymbol{i}=1,2,3,4,5,6,7\right\} . \boldsymbol{\delta}$ and $\boldsymbol{\delta}^{\prime}$ are the general changes of input controlling parameters, which means $\boldsymbol{k}$ times of 0.02 (or 0.2 for CRITICAL SLOPE only) added to the reference values. $\boldsymbol{\Delta}$ is the general change of PBFs caused by $\boldsymbol{\delta}$ or $\boldsymbol{\delta}^{\prime}$.

First-order difference to fixed reference $\left(\boldsymbol{P} \boldsymbol{B} \boldsymbol{F}_{\mathbf{1}}^{\boldsymbol{k}}\right)$ : its role is to illustrate the recommended location of initial default value on the $\mathbf{X}$ axis.

First-order difference to shifting reference $\left(\boldsymbol{P} \boldsymbol{B} \boldsymbol{F}_{2}^{\boldsymbol{k}}\right)$ : its role is to illustrate the adjacent relationship of two locations on the $\mathbf{X}$ axis.

\subsection{Image Metrics}

To evaluate the geographical expansion of cities from the predicted imagery, four evaluation indicators are employed: Urban expansion directional distribution, Urban clusters aggregation, Urban area proportion, and Roads' correlation with urban area (Table 1).

\subsection{AHP method}

With the analytic hierarchy process (AHP), attributed weight of $\left\{\boldsymbol{X}_{i}\right\}$ based feedback model was calculated. A specific description about the weight determination method was given by Thomas L. Saaty (2013) (Saaty, 2013). From stage (2), the bottom-up quantitative relationship between the model input SMP variables and the model output imagery indices could be established as follows:

$$
N \times X=M
$$

where $N$ is the model input variable groups of $\left\{N_{i}, i=1 \ldots 6\right\}$ and $\boldsymbol{M}$ is the model output imagery index groups of $\left\{\boldsymbol{M}_{\boldsymbol{j}}, \boldsymbol{j}=1 \ldots 4\right\} . \boldsymbol{X}$ stands for the transition matrix from SMP to imagery indices, which is used to explain the quantitative transition mechanism of stage (2).

In the quantitative transition mechanism of stage (3), according to the difference between the predicted imagery and the real one, the desired $\boldsymbol{\delta}$ alteration added upon the $\boldsymbol{N} \boldsymbol{i}$ parameters could be calculated. The stage (3) applied the prejudging experience established in the stage (1) and stage (2), to predict a fitting imagery that is close to the real one.

An example of the $\left\{\boldsymbol{X}_{i}\right\}$ as below is calculated.

$X=N^{-1} M=\left[\begin{array}{lllllll}N_{1} & N_{2} & N_{3} & N_{4} & N_{5} & N_{6} & N_{7}\end{array}\right]^{-1}\left[\begin{array}{llll}M_{1} & M_{2} & M_{3} & M_{4}\end{array}\right]$ (4) 


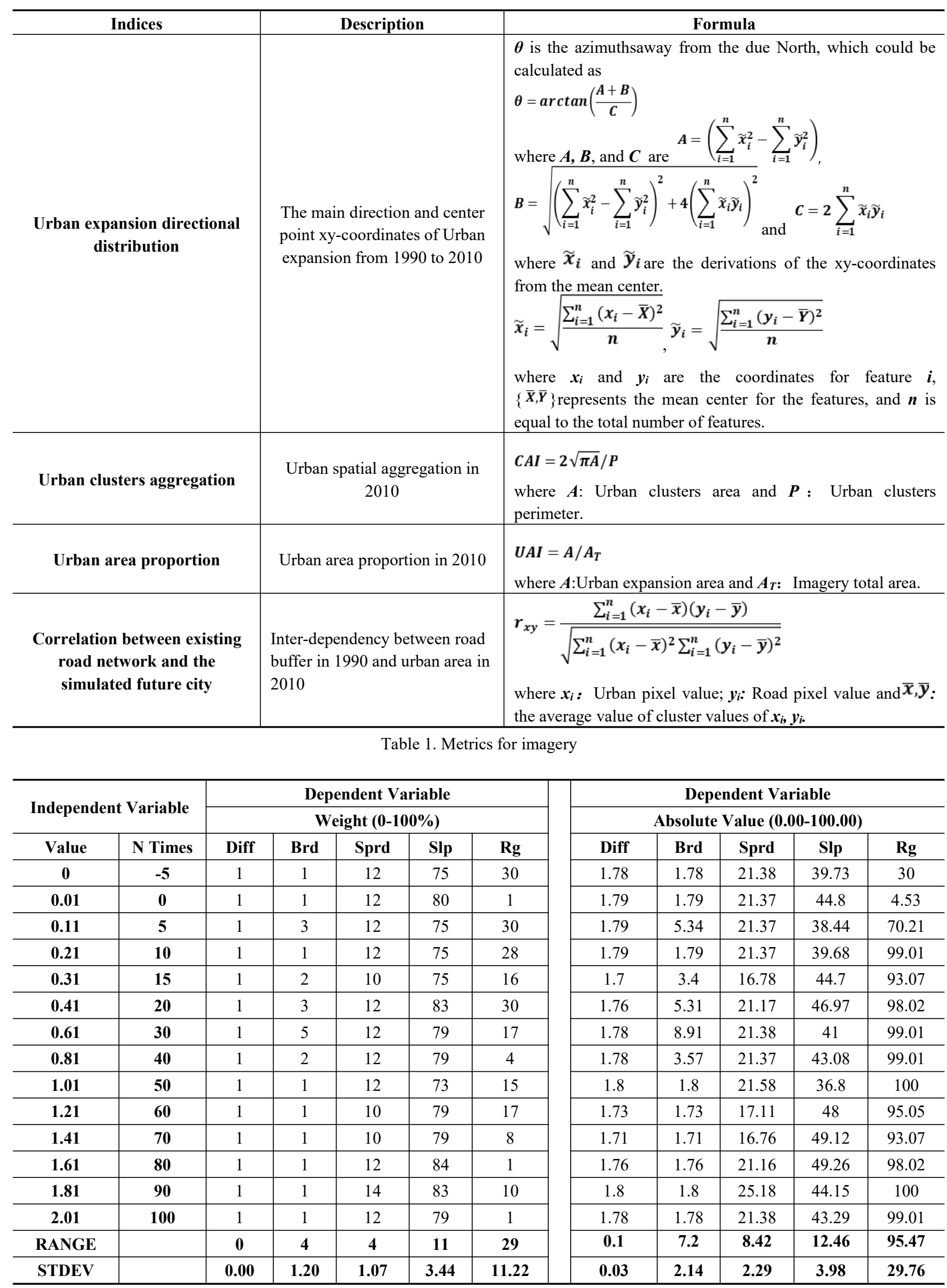

Table 2. Road_grav_sensitivity index series variations and the corresponding model PBF responses 


\section{RESULTS}

To detect the sensitivity in details, different relationship expression strategies are applied: 1) weight; 2) absolute value; 3) first-order difference of the absolute value using the fixed initial default value; and 4) first-order difference of the absolute value using shifting adjacent values. To detect the SA details, a quantitative analysis of the procedural record, final graphic and data results is carried out. Two research tasks were performed.

\subsection{The priority sorting of SMPs}

The model PBF responses to the SMP variations consist of the following data: Weight $(0-100 \%)$ and Absolute value $(0.00$ 100.00). Table 2 shows the variation of each group of input model parameters and the corresponding model PBF responses. Table2 illustrates stage (1) in figure 2, that is, the variations of six groups of input model parameters and the corresponding model PBF responses, including road_grav_sensitivity, slope_sensitivity, critical_low, critical_high, critical_slope, boom and bust. The interval of the independent variable is set to be a regular pattern, and the fine distinctions caused by the variation away from the model initialization parameters A-F are studied.

The First-Order Difference to a Fixed Reference is used to reflect where the initial default value is properly located on the $\mathbf{X}$ axis, while the First-Order Difference to a Shifting Reference method is employed to judge the adjacent relationship of two locations on the $\mathbf{X}$ axis (Figure 4).
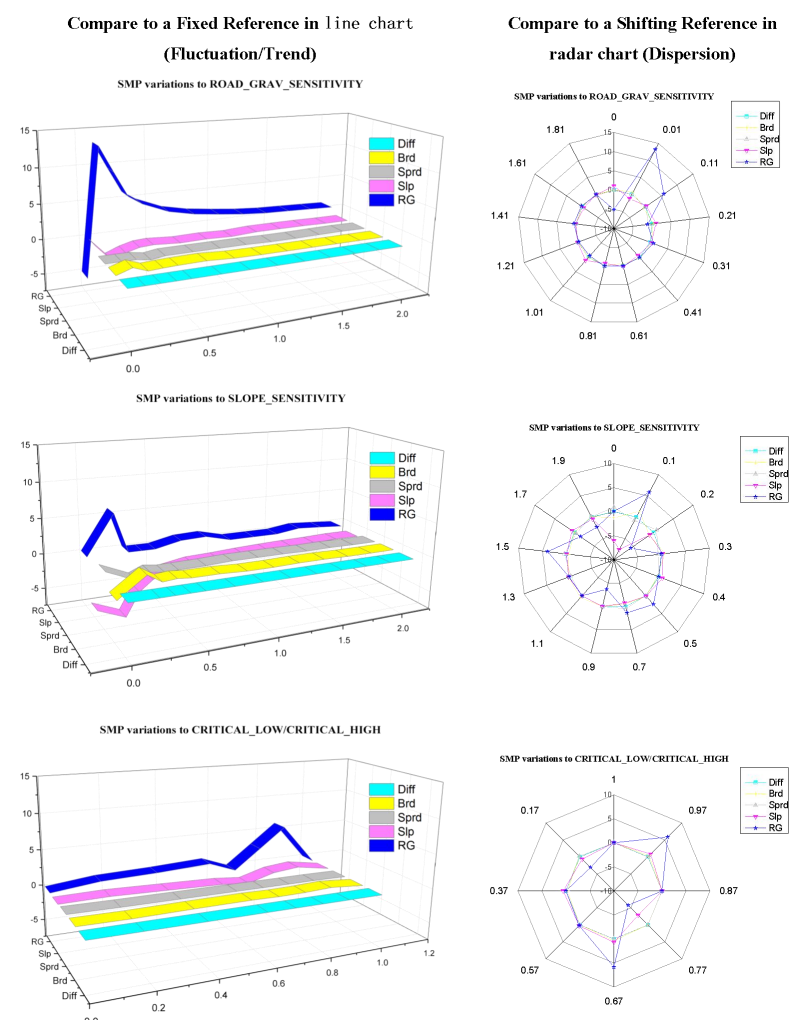

Figure 4. SMP indices variations and the corresponding model PBF response in different forms

In Figure 4, when the SA details of stage (1) are explored, two chart forms of the First-Order Difference evaluating SMP indices variations are employed: line chart and radar chart. Line chart is applied to explore the rules of trend and fluctuation. As a supplement, radar chart is a form of a two-dimensional chart used to observe the dispersion of multivariate data and detect the outliers.

\begin{tabular}{|c|c|c|c|c|c|}
\hline \multirow{2}{*}{} & \multicolumn{5}{|c|}{ Dependent Variable } \\
\hline & \multicolumn{5}{|c|}{ Weight (0-100\%) } \\
\hline $\begin{array}{c}\text { Sensitivity degree } \\
\text { (\%) }\end{array}$ & Diff & Brd & Sprd & Slp & Rg \\
\hline $80-100$ & & & & & \\
\hline $60-80$ & & & & $\sqrt{ }$ & $\sqrt{ }$ \\
\hline $40-60$ & & & & & \\
\hline $20-40$ & & $\sqrt{ }$ & & & \\
\hline 0-20 & $\sqrt{ }$ & & $\sqrt{ }$ & & \\
\hline Average STDEV & 1.62 & 4.21 & $\mathbf{2 . 8 8}$ & 13.05 & 15.99 \\
\hline Average Range & 4.42 & 13.66 & 8.78 & 44.91 & 38.54 \\
\hline
\end{tabular}

Table 3. Sensitivity Rank of Dependent Variables' Weight

From Table 3, the Slp and Rg responsed the parameter variation greatly. Table 3 summarizes their behaviour and ranks them by sensitivity degree, which explains how much the SMPs' variation of $\boldsymbol{\delta}$ contributes to the alteration of $\boldsymbol{\Delta}$ in Weight form.

From Table 3, the weight ranking of the SMPs for all criteria is as follows: $(\mathrm{Slp}, \mathrm{Rg})>\mathrm{Brd}>(\mathrm{Diff}, \mathrm{Sprd})$. Slp and $\mathrm{Rg}$, which are assigned weights of $60 \% \sim 80 \%$, are high-sensitivity indices. Similarly, Brd is a low sensitivity index, while Diff and Sprd are extremely low sensitivity indices.

\subsection{Imagery Responses to SMP variations}

In this part, predicted output imagery and its imagery description indices are discussed, to explore the stage (2). Because there are too many predicted output images, $20 * 65$, simplified metrics are used to measure the charts. They are six groups of input model parameters variations. The response results include Urban expansion directional distribution, Urban clusters aggregation, Urban area proportion, and Roads' correlation with urban area, respectively (Table 1).

Figure 5(a) - (d) illustrate the corresponding model imagery metrics' responses with the variation of model SMPs .

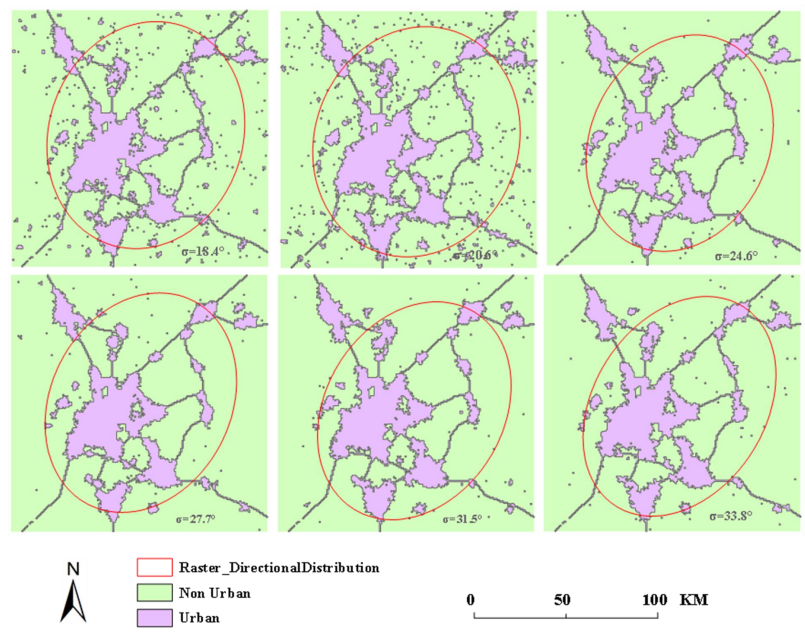

Figure 5(a). Imagery metrics response1: Urban expansion 


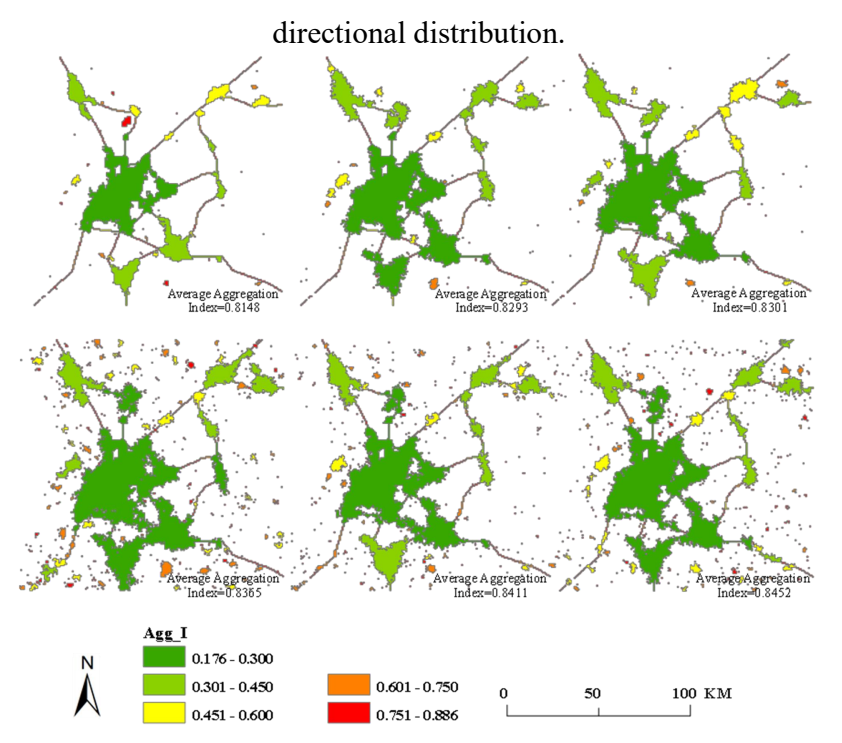

Figure 5(b). Imagery metrics response2: Urban clusters aggregation.

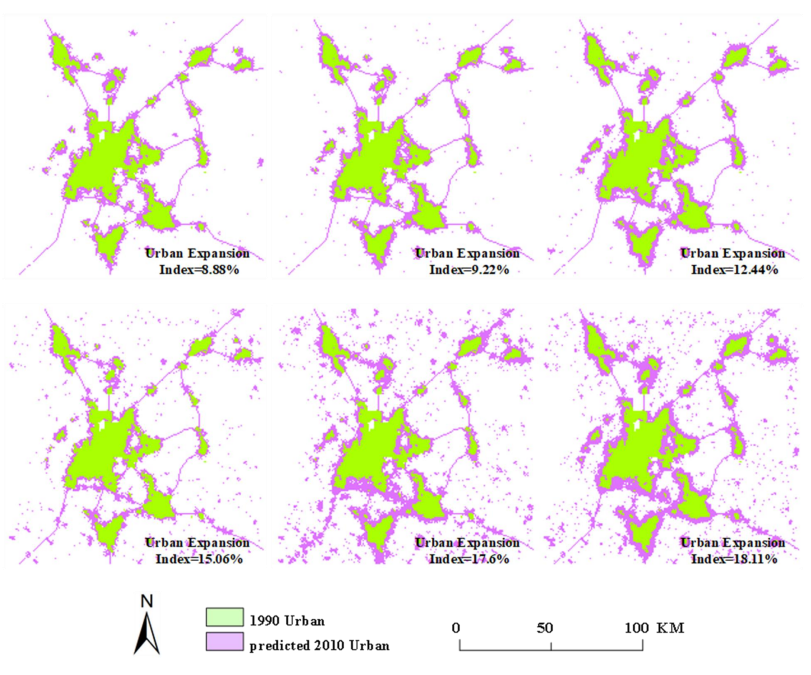

Figure 5(c). Imagery metrics response3: Urban area proportion.

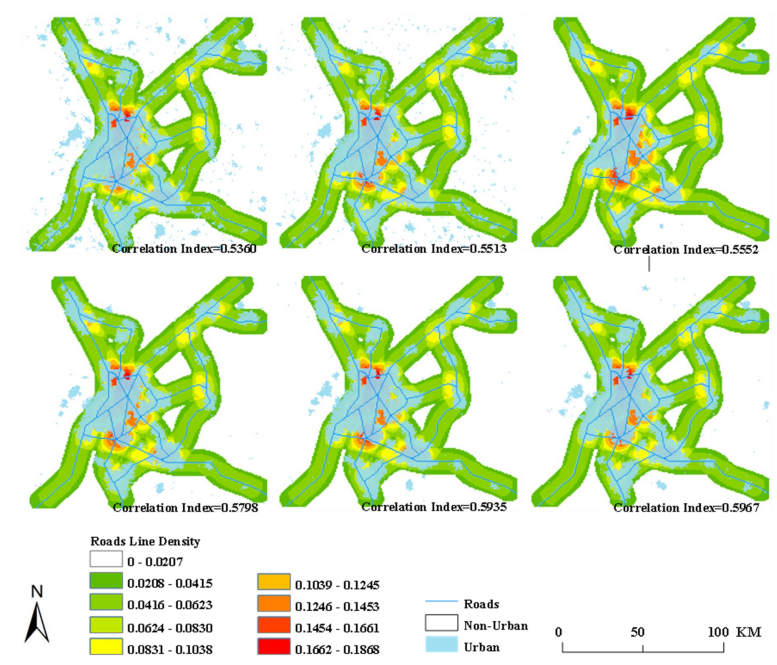

Figure 5(d). Imagery metrics response4: Roads' correlation with urban area.

\subsection{Parameters adjusted with AHP method}

Using MATLAB, an example of the $\left\{\boldsymbol{X}_{i}\right\}$ as below is calculated. With the analytic hierarchy process (AHP), attributed weight of $\left\{\boldsymbol{X}_{\boldsymbol{i}}\right\}$ based feedback model was calculated. The weights of seven factors $\boldsymbol{X}_{1}-\boldsymbol{X}_{7}$ are calculated by eigenvectors of corresponding characteristic roots that are at the maximum. By calculating the consistency checks value $(\mathrm{CK})$, the maximum eigenvalue (CI) and the consistency ratio (CR) are obtained as 4, -0.5 and 0.006 . When the consistency ratio (CR) is less than 0.01 , the rationality of consistency and weight can be accepted (Equation (5)).

$$
\text { We ight of } X=\left[\begin{array}{l}
W_{x 1} \\
W_{x 2} \\
W_{x 3} \\
W_{x 4} \\
W_{x 5} \\
W_{x 6} \\
W_{x 7}
\end{array}\right]=\left[\begin{array}{c}
0.11 \\
0.14 \\
0.31 \\
0.32 \\
0.11 \\
0 \\
0.01
\end{array}\right]
$$

\section{DISCUSSION AND CONCLUSIONS}

In this research, based on the combination of different SMPs settings, the parameter-result record database is obtained. It helps to offer prejudging knowledge between SMPs and outcome images, in both the adjusting size and its sign. The meaning and value of the proposed work is shown in below five aspects.

(1) The exploration study of the transmission mechanism of process-driven model is meaningful; nevertheless, it is a timeconsuming task. Take the SLEUTH model into consideration, the accumulative machine calculation time takes 11 hours for a $200 \times 200$ pixels' image, not to mention larger remote sensing images. The exploration study of the transmission mechanism of SLEUTH model seems to be possible only by adopting the easiest method. Although it is the fact that the morphology and structure of urban prediction sprawl results vary greatly owing to the self-modification parameters (SMPs) setting, the timeconsuming defect of SLEUTH model will skip the validation and verification procedure normally. Generally speaking, the default SMPs values are adopted, rather than the proper parameters group which would predict images close to the real ones.

(2) Measure the weight of the SMPs, and put them in descending order. That is what we have done in this research paper. It is necessary to carry out the sorting work according to the influence weight of the SMPs to the results. Assuming that the prediction results deviated from the real urban expansion results, and that the prediction results still continue the law of historical growth, which factor should we should adjust, the critical_slope parameter or the road_grav_sensitivity parameter. The parameter weight sorting job carried out in this study provides a prejudging experience for parameter adjusting priority in the later stage.

(3) Not only the parameter type selected for changing model operation results should be given attention to, but the value for a certain specific parameter also affects model operation results. For example, should we set the critical_slope parameter to 15 , 17 or 25 ? We need to record the fluctuation size and trend before we can modify the initial parameters of the model. 
(4) The example data of $200 * 200$ pixels and $0-7$ types of ground objects has been used for construction of the experience knowledge base. While there is no standard remote sensing image, considering for other remote sensing imagery with different spatial scale and components, the parameter-result rule could be well applied equally. Because the built-in drive mechanism of the model is fixed, so the input parameter strategy could be applied to any other object when it comes to change the trend and size of the results.

(5) About how to deduce the reasonable initial SMPs values the model needed, this study also describes an AHP training algorithm with adaptive control, using MATLAB. Its architecture is determined in global optimization by patterngenetic algorithm.

To conclude, the transmission mechanism procedures for the proper self-modification parameters setting suggest the following improvements to the development of SLEUTH models.

In the forward stage (1), two derivatives from the Absolute Value are developed, First-order difference to fixed reference ( $\theta_{1}^{i}$ )and First-order difference to shifting reference $\left(\theta_{2}^{i}\right)$, to check the proper locations and two adjacent relationship of two locations on the $\mathbf{X}$ axis. Based on the above two derivatives, three points could be drawn: a) the biggest contributors are screened out and sorted in descending order according to the weight values (Table 4); b) whether the initial default value setting is the appropriate one for each index is evaluated (Table 5 ); and c) the volatility features of a trend are described by the absolute value curve (Figure 5).

In the forward stage (2), four imagery evaluation indicators are employed, Urban expansion directional distribution, Urban clusters aggregation, Urban area proportion and Roads' correlation with urban area as urban morphology quantifies metrics. They could perform good quantitative evaluation role of images' discrepancy (Figure 5).

In the reverse stage (3), based on the SA prejudged default reservoir, the transition mechanism could be expressed as a matrix $\mathbf{X}$.

With the above contributions, an important Feedback mechanism was extracted from the geo-simulation process, and the weights of $\boldsymbol{X}_{1}-\boldsymbol{X}_{7}$ could be drawn (equation (5)).

This process could supply a routine when applying this framework for analysing other models' inherent operational parameter-result relationship. Moreover, global SA shows more efficiency, accuracy and robustness, so its use is proposed for future research. Furthermore, a multiple sampling method is a promising way for the further development of the proper model parameters setting (e.g., a two-level fractional factorial screening method or deriving-based global sensitivity method).

\section{ACKNOWLEDGEMENTS}

This work was supported by the Major State Basic Research Development Program of China [2015CB954101], National Natural Science Foundation of China [41601150], Beijing Key Laboratory of Urban Spatial Information Engineering [2018205] and Fundamental Research Funds for Beijing Universities of Civil Engineering and Architecture[X18092].
Data and Software are supported by SLEUTH beta 3.0 by Project Gigalopolis.

\section{REFERENCES}

Beven, K., Binley, A., 1992. The future of distributed models: model calibration and uncertainty prediction. Hydrological processes $6,279-298$.

Beven, K.J., 2011. Rainfall-runoff modelling: the primer. John Wiley \& Sons.

Chaudhuri, G., Clarke, K., 2013. The SLEUTH land use change model: A review. Environmental Resources Research 1, 88-105.

Chaudhuri, G., Foley, S., 2019. DSLEUTH: a distributed version of SLEUTH urban growth model, 2019 Spring Simulation Conference (SpringSim). IEEE, pp. 1-11.

Clarke, K.C., 2008. Mapping and modelling land use change: an application of the SLEUTH model, Landscape analysis and visualisation. Springer, pp. 353-366.

Feng, H., Liu, H., Zhou, B., Mao, X., Zhao, X., 2012. Study on the Parameters Behavior of the SLEUTH Model. Geography and Geo-Information Science, 12.

Hewitt, R., Diaz-Pacheco, J., 2017. Stable models for metastable systems? Lessons from sensitivity analysis of a Cellular Automata urban land use model. Computers, Environment and Urban Systems 62, 113-124.

Jafarnezhad, J., Salmanmahiny, A., Sakieh, Y., 2016. Subjectivity versus objectivity: comparative study between brute force method and genetic algorithm for calibrating the SLEUTH urban growth model. Journal of Urban Planning and Development 142, 05015015.

Jat, M.K., Choudhary, M., Saxena, A., 2017. Urban growth assessment and prediction using RS, GIS and SLEUTH model for a heterogeneous urban fringe. The Egyptian Journal of Remote Sensing and Space Science 10, 1-19.

Kocabas, V., Dragicevic, S., 2006. Assessing cellular automata model behaviour using a sensitivity analysis approach. Computers, Environment and Urban Systems 30, 921-953.

Park, J., Yang, G., Satija, A., Scheidt, C., Caers, J., 2016. DGSA: A Matlab toolbox for distance-based generalized sensitivity analysis of geoscientific computer experiments. Computers \& Geosciences 97, 15-29.

Saaty, T.L., 2013. The modern science of multicriteria decision making and its practical applications: The AHP/ANP approach. Operations Research 61, 1101-1118.

Saltelli, A., Ratto, M., Andres, T., Campolongo, F., Cariboni, J., Gatelli, D., Saisana, M., Tarantola, S., 2008. Global sensitivity analysis: the primer. John Wiley \& Sons.

Saxena, A., Jat, M.K., 2019. Capturing heterogeneous urban growth using SLEUTH model. Remote Sensing Applications: Society and Environment 13, 426-434. 
Saxena, A., Jat, M.K., 2020. Land suitability and urban growth modeling: Development of SLEUTH-Suitability. Computers, Environment and Urban Systems 81, 101475.

Schouten, M., Verwaart, T., Heijman, W., 2014. Comparing two sensitivity analysis approaches for two scenarios with a spatially explicit rural agent-based model. Environmental Modelling \& Software 54, 196-210.

Shafizadeh-Moghadam, H., Asghari, A., Tayyebi, A., Taleai, M., 2017. Coupling machine learning, tree-based and statistical models with cellular automata to simulate urban growth. Computers, Environment and Urban Systems 64, 297-308.

Smith, D.O., 2019. Third world cities in global perspective: the political economy of uneven urbanization. Routledge.

Song, X., Zhang, J., Zhan, C., Xuan, Y., Ye, M., Xu, C., 2015. Global sensitivity analysis in hydrological modeling: Review of concepts, methods, theoretical framework, and applications. Journal of hydrology 523, 739-757.

USGS, UCSB, 2005. SLEUTH3.0beta_p01.

Xu, E., Zhang, H., 2013. Spatially-explicit sensitivity analysis for land suitability evaluation. Applied Geography 45, 1-9.

Zoras, S., Triantafyllou, A., Hurley, P., 2007. Grid sensitivity analysis for the calibration of a prognostic meteorological model in complex terrain by a screening experiment. Environmental Modelling \& Software 22, 33-39. 